\title{
Design and performance verification of a microscope-based interferometer for miniature-specimen metrology
}

\author{
Chih-Kung Lee, MEMBER SPIE \\ National Taiwan University \\ Institute of Applied Mechanics \\ Taipei 106, Taiwan \\ E-mail: cklee@ ntu.edu.tw
}

\author{
Wen-Jong Wu, MEMBER SPIE \\ National Taiwan University \\ Department of Engineering Science and \\ Ocean Engineering \\ Taipei 106, Taiwan
}

\section{Giin-Yuan Wu}

Sunwave Optoelectronics, Inc. 4F, No. 258 Lian-Cheng Road

Chung-ho, Taipei hsien 235, Taiwan

\author{
Chia-Ling Li \\ Zeng-De Chen \\ Jau-Yu Chen \\ National Taiwan University \\ Institute of Applied Mechanics \\ Taipei 106, Taiwan
}

\begin{abstract}
Design and experimental verification of a microscope-based circular-polarization interferometer developed for measuring the vibration of miniature specimens is detailed. This interferometer, which comes in either a two-detector or a four-detector configuration, demonstrates that the optimum operating point of a circular-polarization interferometer depends strongly on the signal detection and processing algorithms adopted. The influence of the specimen surface optical properties on the desired operating conditions is also examined. The optimum operating point of the two-detector configuration demands that the two returning light beams emitting from the specimen possess equal intensity, which matches the understanding of traditional interferometers, where two equal interference arms achieves the best interference efficiency. Surprisingly, it appears that the optimum intensity ratio for the four-detector configuration occurs if the two interference arms possess equal intensity before it hits the specimen. That is, under the constraint of a constant light source, it appears that the optimum operating point of a fourphotodetector circular-polarization interferometer is independent of the surface optical properties. Both theoretical and experimental results that verify the interaction between the optoelectronic configuration and the signal-processing algorithms implemented are presented. () 2005 Society of Photo-Optical Instrumentation Engineers. [DOI: 10.1117/1.2010147]
\end{abstract}

Subject terms: optomechanical design; signal processing; laser Doppler velocimetry; interferometry; metrology; velocimetry.

Paper 040352R received Jun. 5, 2004; revised manuscript received Dec. 23 , 2004; accepted for publication Feb. 7, 2005; published online Aug. 10, 2005.

\section{Introduction}

Laser Doppler interferometers and vibrometers have long been widely accepted as practical and effective tools for both displacement and velocity analysis of precision mechanical systems. With the rapid performance improvement of ultrahigh-precision mechanical systems such as microelectromechanical systems, we observe that optical and magnetic disk drives, compact disk drives, and digital video disk drives, sensors, and actuators have all been dramatically reduced in size to increase mechanical performance. Attempting to catch up with this trend, metrology systems used to characterize the performance of these highperformance mechanical systems have decreased in size and at the same time improved in performance. When such optical systems are used, the magnitude of the object velocity or displacement can be obtained directly by measuring the Doppler frequency or the corresponding phase. However, the direction of the object motion cannot be discerned by such a simple measurement, for negative frequency that corresponds to motion along (say) the negative direction, will be measured to be positive, because the optical frequency shift is typically measured by optical inter-

0091-3286/2005/\$22.00 @ 2005 SPIE ference. This phenomenon, termed directional ambiguity, ${ }^{1-4}$ is typically overcome by adopting a frequency-shift or circular-polarization technique.

The frequency-shift configuration essentially introduces a frequency shift into one of the light beams utilized, which can be achieved by using an acoustic-optical modulator, moving grating, or other method., ${ }^{3,5}$ The second configuration, which is equally useful, is called a circularpolarization interferometer. ${ }^{6-12}$ This technology does not require any phase-shift or frequency-shift devices within the system and presents us with the possibility of creating a Doppler metrologyy system, which is smaller and more compact. The microscope-based interferometer presented in this paper utilizes a circular-polarization configuration due to its design size specifications.

It is well known that the range and resolution of interferometers and vibrometers are strongly affected by the optical system design and signal-processing method adopted. We show in this paper a new technique that can significantly influence the design configuration of interferometers and vibrometers, for the optimum operating condition of circular polarization interferometers is in fact strongly influenced by the signal-processing algorithms used.

\section{Theory}

As mentioned, a circular-polarization interferometer configuration was adopted to develop a microscope-based in- 


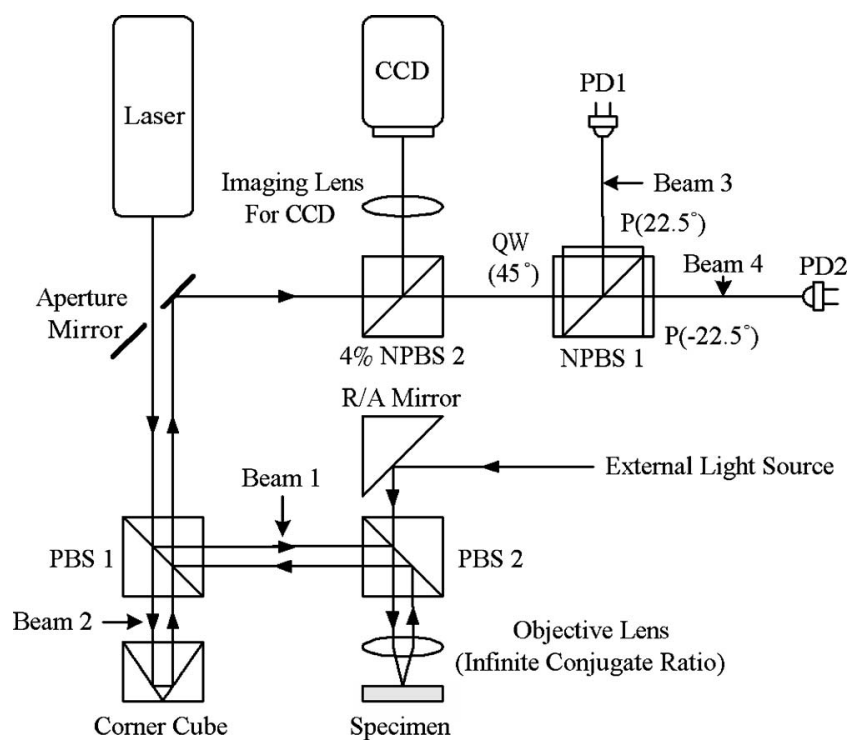

Fig. 1 Schematic of a two-photodetector interferometer-vibrometer.

terferometer in order to facilitate the metrology of miniature specimens typically found in today's high-tech applications. The basic idea was to adopt a microscope with an infinite-conjugate-ratio objective as the basis of the integration. For this type of microscopic objective, ${ }^{13}$ a collimated light beam propagating at the back of the plane will focus onto the specimen, which provides us with a platform to integrate any additional subsystems having no optical power directly within the optical path formed by the objective and the eyepiece.

To demonstrate the strong interaction between the optimum operating condition and the interferometer design, two types of microscope-based advanced vibrometer interferometer devices, ${ }^{5,7-9}$ with the acronym AVID, were developed (Figs. 1 and 2) with addition of a small polarization beamsplitter into the imaging path of the microscope.

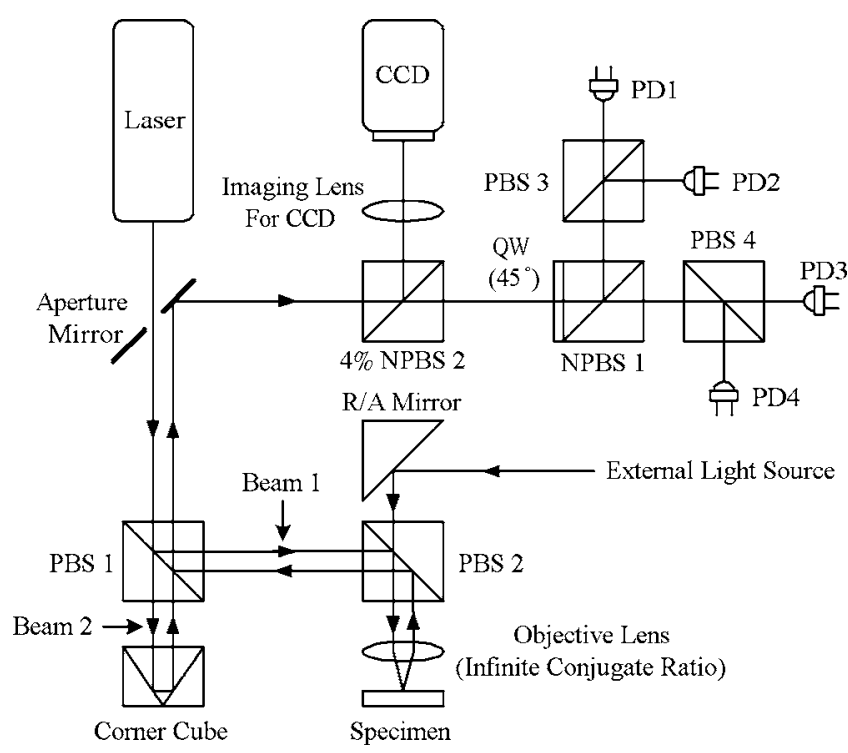

Fig. 2 Schematic of a four-photodetector interferometer-vibrometer.
The two-photodetector configuration (AVID 2) is shown in Fig. 1, and the four-photodetector configuration (AVID 4) is shown in Fig. 2. The only difference between these two configurations is the number of photodetectors placed behind the two polarization beamsplitters instead of the two photodetectors located behind the two linear polarizers that are used for signal detection. The emitted light beam is split into beam 1 and beam 2 with a polarization beamsplitter, PBS1. After splitting, the two outgoing beams have electric field amplitudes $E_{10}$ and $E_{20}$, and optical intensities $I_{10}$ and $I_{20}$, respectively. Beam 1 propagates back and forth from the specimen and carries the specimen displacement and velocity information. On the other hand, beam 2 transmits and reflects from a corner cube to serve as the reference arm in both AVID 2 and AVID 4. Because a microscopic objective lens of infinite conjugate ratio (objective lens in Figs. 1 and 2) is used to focus beam 1 onto the specimen, the system can perform an interferometric measurement regardless of whether the specimen is specular or diffusive. Nevertheless, it can be shown that the surface properties of the specimen do influence the optimum operating point, depending on whether the AVID 2 configuration or the AVID 4 configuration is adopted. An external light source, such as a mercury arc lamp, is required to illuminate the specimen so the image on the CCD is visible.

The outgoing light beam 1 enters the microscope objective slightly off center with respect to the optical axis of the objective (Figs. 1 and 2) in order to introduce the desired offset. Similarly, the outgoing light beam 2 enters the corner cube off center with respect to the tip of the corner cube, so as to create an offset of the same magnitude. With the offset mentioned, the returning beams get reflected by the aperture mirror and propagate toward the 4\% NPBS2 (nonpolarizing beamsplitter). The $4 \%$ returning light beams reflected by NPBS2 form a bright spot on the CCD, which is used to fix the location of the measurement point, which, coupled with the specimen image illuminated by the external light source, provides a useful tool for setting the measurement spot. It should be noted that the polarization states of these two returning light beams are orthogonal to each other right before hitting the quarter-wave plate $\mathrm{QW}\left(45^{\circ}\right)$, i.e., the two light beams will not interfere. This is the standard configuration required by the circular-polarization interferometer $^{7-12}$ mentioned. The two returning light beams transmitted through NPBS2 are incident onto QW $\left(45^{\circ}\right)$, which has its fast axis inclined at $45 \mathrm{deg}$ to the electric fields of the two returning light beams. After passing $\mathrm{QW}\left(45^{\circ}\right)$, the two returning beams are converted into a right and a left circularly polarized beam, respectively. Expressed as Jones vectors, ${ }^{14}$ the electric vector fields of the two returning light beams 1 and 2 can be written as

$$
\begin{aligned}
& \mathbf{E}_{1}=A_{1} E_{10}\left[\begin{array}{l}
1 \\
i
\end{array}\right] \exp \left[i 2 \pi\left(f_{l}+f_{d 1}\right) t\right], \\
& \mathbf{E}_{2}=A_{2} E_{20}\left[\begin{array}{c}
1 \\
-i
\end{array}\right] \exp \left[i\left(2 \pi\left(f_{l}+f_{d 2}\right) t+\phi\right)\right],
\end{aligned}
$$

where $i=\sqrt{-1}, f_{l}$ is the laser initial frequency, $f_{d 1}$ and $f_{d 2}$ are the Doppler frequencies caused by the motion of the object, $\phi$ is the initial phase difference of these two light 
beams, and $A_{1}$ and $A_{2}$ are the ratios between the returning electric field amplitude and the incident light in beam 1 and beam 2 . It should be noted that the transmittance or reflectivity of all the components influences these two ratios. Because a corner cube was used to reflect light beam $2, f_{d 2}$ equals zero, but it is kept in the theoretical derivations herein for generality. Combining these two returning light beams leads to a linearly polarized light beam, the azimuth of whose electric field vector depends on the relative phase of the two returning light beams. We can see that

$$
\begin{aligned}
\mathbf{E}= & \left\{A_{1} E_{10}\left[\begin{array}{l}
1 \\
i
\end{array}\right] \exp \left[i 2 \pi f_{d 1} t\right]+A_{2} E_{20}\left[\begin{array}{c}
1 \\
-i
\end{array}\right]\right. \\
& \left.\times \exp \left[i\left(2 \pi f_{d 2} t+\phi\right)\right]\right\} \exp \left[i 2 \pi f_{l} t\right],
\end{aligned}
$$

$I=\mathbf{E} \cdot \mathbf{E}^{*}=I_{1}+I_{2}+2 \sqrt{I_{1} I_{2}} \cos \left[2 \pi\left(f_{d 2}-f_{d 1}\right) t+\phi\right]$,

where $I$ is the combined light intensity, and $I_{1}=A_{1}^{2} I_{10}$ and $I_{2}=A_{2}^{2} I_{20}$ are the intensities of the two returning light beams 1 and 2 , respectively. In addition, $I_{10}+I_{20}$ equals the laser intensity and is assumed to be a constant. A nonpolarization beamsplitter (NPBS1) splits the combined returning light beams further for processing.

The relative Doppler frequency shift $f_{d}=f_{d 2}-f_{d 1}$ is related to the velocity of motion of the specimen. In brief, the velocity $V$ of the specimen parallel to the laser beam can be obtained by

$f_{d}=\frac{2 V}{\lambda}=\frac{2 V f_{l}}{c}$

where $c$ is the speed of light and $\lambda$ is the wavelength of the laser beam. Since the time-dependent phase variation $\Phi$ is produced by the displacement $D$ of the specimen, the relationship of $\Phi$ and $D$ is

$\frac{\Phi}{2 \pi}=\frac{f_{l}}{c} \cdot 2 D=\frac{f_{l}}{c} \cdot 2 V t+\phi=f_{d} t+\phi$

Hence the displacement $D$ can be determined by measuring the phase variation $\Phi$. That is to say, AVID can measure both velocity and displacement independently by performing frequency demodulation and phase decoding, respectively.

\subsection{Two-Photodetector Configuration}

As was mentioned, AVID 2 utilizes two photodetectors, PD1 and PD2, to perform the measurements as shown in Fig. 1. Two linear polarizers $\mathrm{P}\left(22.5^{\circ}\right)$ and $\mathrm{P}\left(-22.5^{\circ}\right)$, whose pass axes are at $\pm 22.5 \mathrm{deg}$, respectively, are incorporated into two sides of the nonpolarization beamsplitter (NPBS1) to convert the azimuth angle information of the electric vector field $\mathbf{E}$ [Eq. (1)] to obtain intensity information. More specifically, the electric fields of beam 3 and beam 4 possess a $45-\mathrm{deg}$ phase difference, which is a result of the spatial difference of the +22.5-deg and -22.5-deg polarizers. These two intensity signals are typically called $P-Q$ quadrature signals and are associated with dc terms. Denoting the signal detected by PD1 as $P^{(2)}$ and the signal
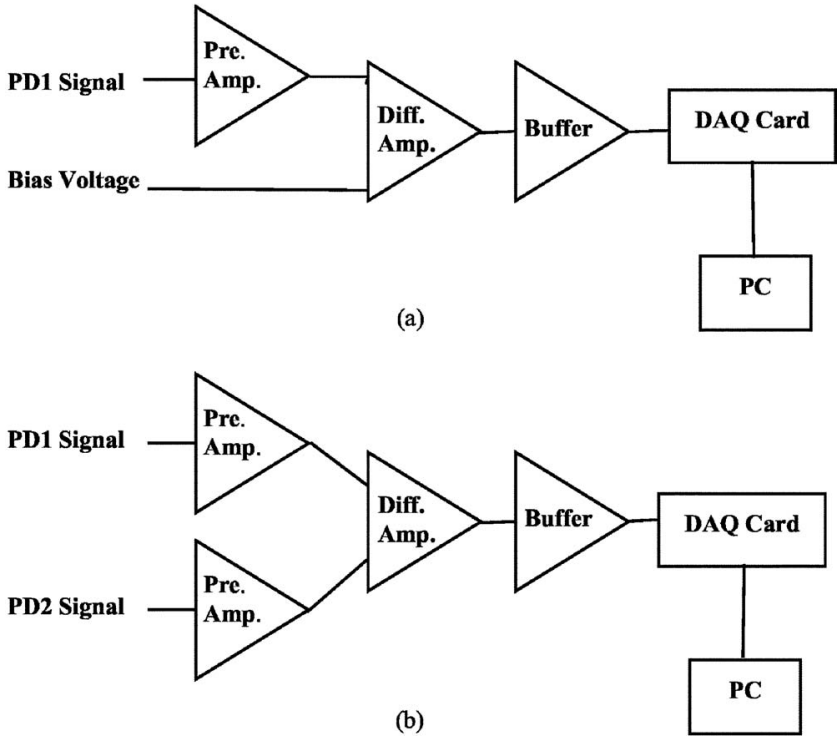

Fig. 3 Electric signal-processing block diagram for (a) AVID 2 and (b) AVID 4

detected by PD2 as $Q^{(2)}$, the phase angle difference of the two intensity signals $P^{(2)}$ and $Q^{(2)}$ has a 90-deg phase difference as shown below:

$$
\begin{aligned}
& P^{(2)} \propto 1+\nu \sin \left[2 \pi\left(f_{d 2}-f_{d 1}\right) t+\phi\right], \\
& Q^{(2)} \propto 1+\nu \cos \left[2 \pi\left(f_{d 2}-f_{d 1}\right) t+\phi\right], \\
& \nu=\frac{2\left(A_{1}^{2} I_{10} \cdot A_{2}^{2} I_{20}\right)^{1 / 2}}{A_{1}^{2} I_{10}+A_{2}^{2} I_{20}},
\end{aligned}
$$

where $0 \leqslant \nu \leqslant 1$ is the visibility of the interference fringe. The physical quantity of interest for the circularpolarization interferometer is a Doppler frequency shift, which is embedded within the ac term of the $P-Q$ quadrature signal as it relates to the moving velocity of the specimen of interest. The $P-Q$ quadrature signal forms a Lissajous circle, and it is clear that the diameter of the circle represents the ac signal amplitude of AVID. In addition, any fuzziness along the diameter direction of the Lissajous circle can be considered as noise. It should also be noted that the visibility $\nu$ is a function of $A_{1}$ and $A_{2}$. Since speckle patterns appear with the returning light beams $A_{1}$ and $A_{2}$ as a result of phase fluctuations induced by the specimen surfaces, the visibility is lower when the specimen surface is optically rough.

Because the dc terms in $P^{(2)}$ and $Q^{(2)}$ saturate the dynamic ranges of the detection circuit, the dc term is typically removed during the processing of the quadrature signal. Figure 3(a) shows a typical circuit used to perform the signal processing, which essentially utilizes an externally supplied bias voltage to subtract the dc term from the photodetector electric signal. In a different measurement case, the bias voltage must be changed according to the specimen optical properties. In this paper, we discuss a case of an 
optimum operating point of a circular polarization interferometer, where the dc term, influenced by the quadrature signal, is eliminated.

For a specific configuration and a fixed set of specimen, $A_{1}$ and $A_{2}$ remain constant during each measurement. The visibility $\nu$ attains its maximum value of 1 when

$A_{1}^{2} I_{10}=A_{2}^{2} I_{20}$,

or

$I_{1}=I_{2}$.

This means that the AVID 2 configuration has the best interference efficiency when the two light beams after hitting the specimen and the corner cube possess identical intensity. Considering the general experimental situation, the corner cube returns almost all the energy of the incident light beam back to the detector. In contrast, the reflectivity of the specimen varies from experiment to experiment. The optimum operating condition shown in Eq. (6) becomes unattainable if the light beam emitted from the laser is split equally by the PBS1. The approach adopted in the design can compensate for this problem by changing the polarization state of the light beam emitted from the laser source by rotation of the laser tube (Fig. 1). More specifically, different incident light intensities must be sent to the corner cube and to the specimen ${ }^{8}$ to compensate for the reflectivity difference of the specimen and corner cube in order to arrive at the optimum condition predicted by Eq. (6).

\subsection{Four-Photodetector Configuration}

The schematic of AVID 4 is shown in Fig. 2. As was mentioned, the only difference between the AVID 2 and AVID 4 configurations lies in the number of photodetectors placed behind the two polarization beamsplitters instead of the two photodetectors located behind the two linear polarizers that are used to measure the optical signals. Like that between PD1 and PD2 in AVID 2 (Fig. 1), the 45-deg phase difference of the electric field between PD1 and PD3 (Fig. 2) is caused by the 45-deg spatial angular difference of PBS3 or PBS4. Similar to the condition under the AVID 2 configuration, the intensity signals of $\mathrm{PD} 1$ and $\mathrm{PD} 3$ possess a 90-deg phase difference, since the intensity is proportional to the square of the electric field amplitude. In addition, a 180-deg phase difference appears between PD1 and PD2 as well as between PD3 and PD4, due to the optical function of the polarization beamsplitter. In summary, the signals detected by these four PD can be expressed as

$I_{1}^{(4)}=I_{1}+I_{2}+2\left(I_{1} I_{2}\right)^{1 / 2} \sin \left[2 \pi\left(f_{d 2}-f_{d 1}\right) t+\phi\right]$,

$I_{2}^{(4)}=I_{1}+I_{2}-2\left(I_{1} I_{2}\right)^{1 / 2} \sin \left[2 \pi\left(f_{d 2}-f_{d 1}\right) t+\phi\right]$,

$I_{3}^{(4)}=I_{1}+I_{2}+2\left(I_{1} I_{2}\right)^{1 / 2} \cos \left[2 \pi\left(f_{d 2}-f_{d 1}\right) t+\phi\right]$,

$I_{4}^{(4)}=I_{1}+I_{2}-2\left(I_{1} I_{2}\right)^{1 / 2} \cos \left[2 \pi\left(f_{d 2}-f_{d 1}\right) t+\phi\right]$.

The two modified signals $P^{(2)}$ and $Q^{(2)}$ obtained from $I_{1}^{(4)}$ $-I_{2}^{(4)}$ and $I_{3}^{(4)}-I_{4}^{(4)}$ can then be derived as

$$
\begin{aligned}
& P^{(4)} \propto M \sin \left[2 \pi\left(f_{d 2}-f_{d 1}\right) t+\phi\right], \\
& Q^{(4)} \propto M \cos \left[2 \pi\left(f_{d 2}-f_{d 1}\right) t+\phi\right], \\
& M=\left(I_{1} I_{2}\right)^{1 / 2}=\left(A_{1}^{2} I_{10} \cdot A_{2}^{2} I_{20}\right)^{1 / 2},
\end{aligned}
$$

where $M$ is the interference fringe amplitude. The dc term is thus eliminated in the configuration.

Figure 3(b) shows the electronic signal-processing circuit adopted in AVID 4. The signals received from each pair of photodiodes (PD1, PD2) and (PD3, PD4) are sent through a pair of differential amplifiers to remove the dc components in order to maximize the dynamic range of the interfacing electronics. The differential circuit removes the common dc component and doubles the required ac signal component. Therefore the Lissajous circle diameters are usually bigger in AVID 4 than in AVID 2.

Since there is no dc component in AVID 4, the visibility no longer exists, and only the fringe amplitude $M$ is concerned. For a fixed set of specimens, $A_{1}$ and $A_{2}$ are constants during each measurement. Furthermore, the total energy emitted by the laser light source is constant, i.e., $I_{10}$ $+I_{20}$ equals a constant $B$. Replacing $I_{20}$ with $B-I_{10}$ in Eq. (8), the amplitude $M$ achieves its maximum when

$I_{10}=I_{20}=\frac{B}{2}$,

as is seen by the calculation of the derivative

$$
\frac{\partial M}{\partial I_{10}}=\frac{\partial}{\partial I_{10}}\left[A_{1}^{2} I_{10} \cdot A_{2}^{2}\left(B-I_{10}\right)\right]^{1 / 2}=0 .
$$

That is to say, making the incident light intensity of beam 1 and beam 2 identical leads to the optimum condition in the AVID 4 configuration, i.e., the desired operating point is independent of the reflectivity or the optical properties of the specimen. In other words, the polarization state of the light beam emitted from the laser can be fixed for most specimens. It is also clear that the optimum condition mentioned in Eq. (9) cannot be achieved when the surface condition of the specimen surface is too scattered or too absorptive, so that the light beam returned by the specimen is buried within the noise. More specifically, if $I_{1} \approx 0$, then $\left(I_{1} I_{2}\right)^{1 / 2} \approx 0$ in Eq. (8) results in the worst interference efficiency. This is not reasonable. In other words, $M$ will not achieve its maximum when $I_{10}=I_{20}$. More specifically, if a highly diffusive or absorptive specimen is encountered, the optimum condition for the AVID 4 configuration becomes $A_{1}^{2} I_{10}=A_{2}^{2} I_{20}$ or $I_{1}=I_{2}$, which is the same as that of the AVID 2 configuration.

\section{Experimental Setup}

As was discussed, the optimum operating point of a circular-polarization interferometer is a strong function of the number of photodetectors and the signal-processing algorithms adopted. All of these assertions are experimentally verified herein. Both AVID 2 and AVID 4 configurations were constructed and tested. As mentioned, the polarization state of the light beam emitted from the 633.2-nm He-Ne laser source was set by rotating the laser tube (Figs. 1 and 


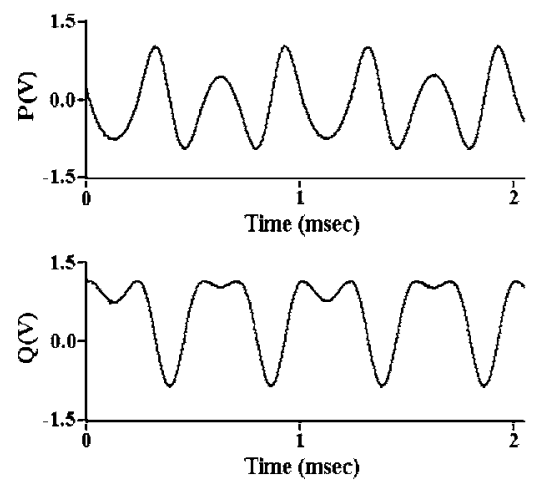

(a)

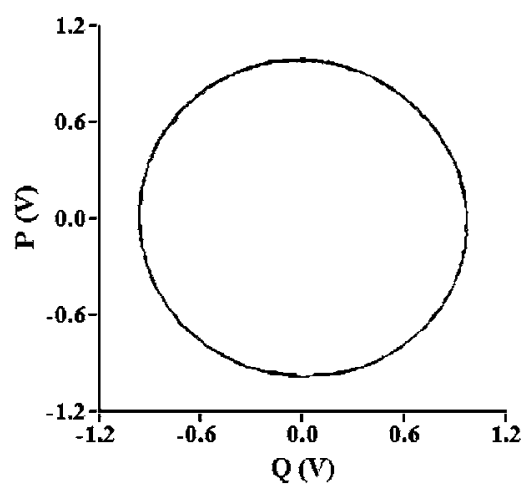

(b)

Fig. 4 AVID measurement results: (a) quadrature signal pair $P, Q$; (b) Lissajous circle pattern.

2). As the light beam passes through the PBS1, the intensity ratio of the two split beams, beam 1 and beam 2 , is then set according to the spatial relationship between PBS1 and the polarization axis of the laser light beam.

Both specular and diffusive surface specimens, such as thin film disks, metal gaskets, and magnets of hard drives, were used to verify the influence of the specimen surface properties at different operating conditions. The thin film disk was found to have a $95 \%$ reflectivity and deemed a specular surface. On the other hand, a magnet only has a $30 \%$ reflectivity and is diffusive in nature, i.e., a magnet is a diffusive specimen. The metal gasket was found to have a $50 \%$ reflectivity and is thus considered a partially reflective surface. All three specimens were mounted on a piece of piezoelectric transducer (PZT) stack, which was driven by a $1-\mathrm{kHz}$ sine wave of $5-\mathrm{V}$ rms amplitude to induce a 500-nm peak-to-peak displacement. The signals detected by the two- and four-photodetector measurement modes were preamplified and differentially amplified by the circuits (Fig. 3) to form the quadrature signal $P$ or $Q$. The only difference is that AVID 2 needs to give a bias voltage according to the specimen surface properties to balance the dc term in Eq. (5), and AVID 4 need not. The data were then downloaded to a computer with a multichannel highspeed simultaneous DAQ card to retrieve the displacement and velocity information of these specimens after some data processing. 5,7

Figure 4 shows two typical representations of the signal obtained from a circular-polarization interferometer, regardless of whether it is an AVID 2 or an AVID 4 configuration. Figure 4(a) presents the data in the $P$ - and $Q$-versus-time format. Plotting the quadrature signal $P$ versus $Q$ leads to the Lissajous circle patterns as shown in Fig. 4(b). Solving for the total phase difference in the Lissajous circle pattern leads to the retrieval of the displacement or velocity of the particular specimen." It is clear from the two data representation formats that the Lissajous circle pattern demonstrates the ac signal amplitude better, in that the fuzziness of the elliptical pattern can be better observed than that of the amplitude-time format shown in Fig. 4(a). All testing data, which compare the ac signal amplitude for different specimens and under different operating conditions, are presented in the Lissajous circle format herein.

\section{Experimental Results and Discussions}

The specular specimen, i.e., the thin film disk, was tested first. Four sets of intensity ratios were implemented in AVID 2 and AVID 4 configurations for this specimen. Several parameters are worth noting when trying to compare the data. The reflectance of the $s$-polarized light and the transmittance of the $p$-polarization light of the PBS adopted in AVID 2 and AVID 4 are very close to $100 \%$. The reflectivity of the corner cube is very close to $100 \%$. The transmittance of the objective lens was experimentally found to be $90 \%$. The reflectivity of the thin film disk was measured to be $95 \%$. Because light beam 1 passes the objective lens twice, the mentioned data lead to the conclusion that the returning beam- 1 intensity is $0.7695\left(=0.9^{2} \times 0.95\right)$ times the outgoing beam-1 intensity for the specular specimen. On the other hand, the intensities of the outgoing and returning beam 2 remain almost identical. Thus, the intensity ratios $4: 1,3: 1,5: 4$, and $1: 1$ of the outgoing beam 1 to beam 2 , correspond to the intensity ratios $3: 1,7: 3,1: 1$, and $3: 4$ of the returning beam 1 to beam 2, shown in Fig. 5(a) and Fig. 5(b), respectively. More specifically, the AVID 4 data shown in Fig. 5 were obtained basically under the same metrology condition as the AVID 2 data.

Since the AVID 4 has no de term and the AVID 2 has a constant dc term, the vertical axis of Fig. 5 uses the diameter to show the fringe amplitude $M$ in AVID 4 and the visibility $\nu$ in AVID 2, which is defined as the average of the long and the short axes of each Lissajous circles. The same goes for Figs. 6 and 7 as well. Comparing the AVID 2 measurement results shown in Fig. 5(a) and Fig. 5(b), it is quite obvious that the Lissajous pattern has the biggest diameter, viz., the maximum ac signal amplitude when the two returning beams have equal intensity. The vertical dashed line identifies the optimum operating condition. This result agrees well with theoretical predictions as shown in Eq. (6). Obtained at the same ratio of outgoing beams $I_{1}$ to $I_{2}$ as that of AVID 2, the AVID 4 measurement data show clearly that the best Lissajous pattern was obtained under the condition where beam 1 and beam 2 are identical in intensity before hitting the specimen. This is different from the AVID 2 measurement data. Comparing the two curves in Fig. 5(a) and Fig. 5(b), the diameters 


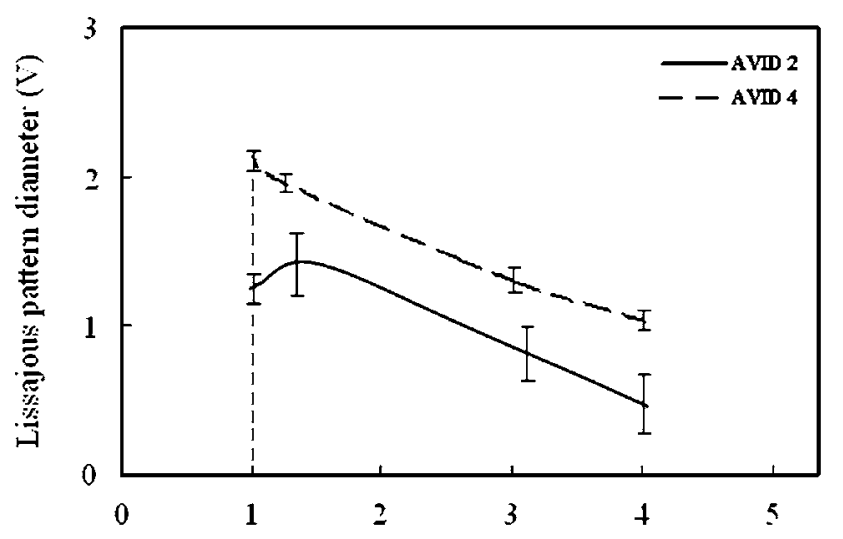

intensity ratio for outgoing beam 1 and beam $2\left(I_{10} / I_{20}\right)$

(a)

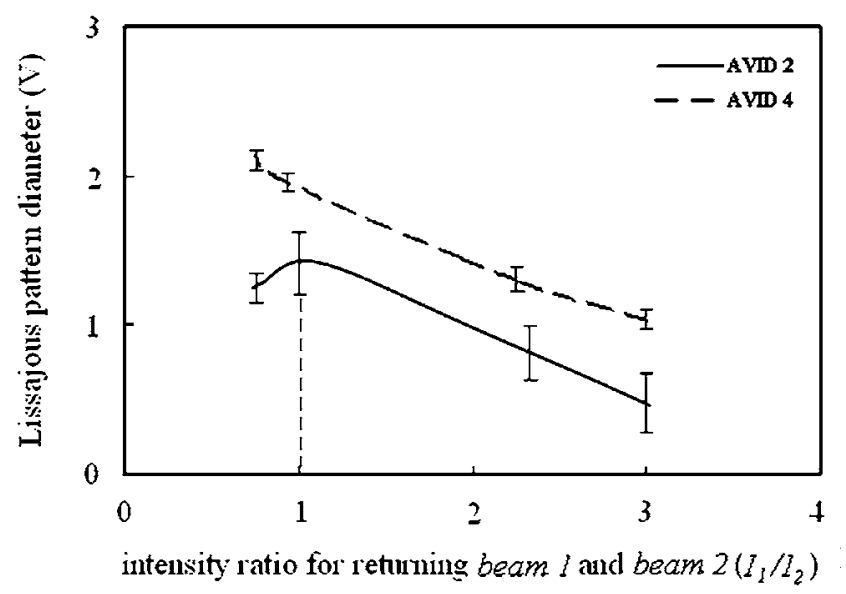

(b)

Fig. 5 Thin film disk (specular surface) measurement results with different intensity ratios for beam 1 and beam 2 expressed in terms of (a) outgoing beams and (b) returning beams. The error bars are the root-mean-square (rms) deviation of the data points from the diameter for the best-fit Lissajous circles and are enlarged 10 times.

from AVID 2 data are generally smaller than those from AVID 4 data. Simultaneously, the error bars from AVID 2 data are larger than those from AVID 4 data. The error bars in Fig. 5 are magnified tenfold; otherwise they could barely be discerned from the thickness of the curves shown. All these experimental data comparisons clearly demonstrate the effect of the differential circuit in AVID 4: it can remove the noise and amplify the $P-Q$ signals. In other words, measuring the same specimen under the same environmental conditions, circular-polarization interferometers equipped with different types of signal detection and processing algorithms can indeed lead to vastly different optimum operating conditions and signal resolutions.

Figure 6 shows the metal gasket measurement results obtained with AVID 2 and AVID 4 under different intensity ratios for beam 1 and beam 2 for (a) outgoing beams and (b) returning beams. The metal gasket measured had a reflectivity of 0.5 , which placed the returning beam- 1 intensity of this case at $0.405\left(=0.9^{2} \times 0.5\right)$ times the outgoing beam-1 intensity. The intensities of the outgoing and returning beam 2 remain almost identical to those of the specular specimen. Even though the surface was more diffusive than

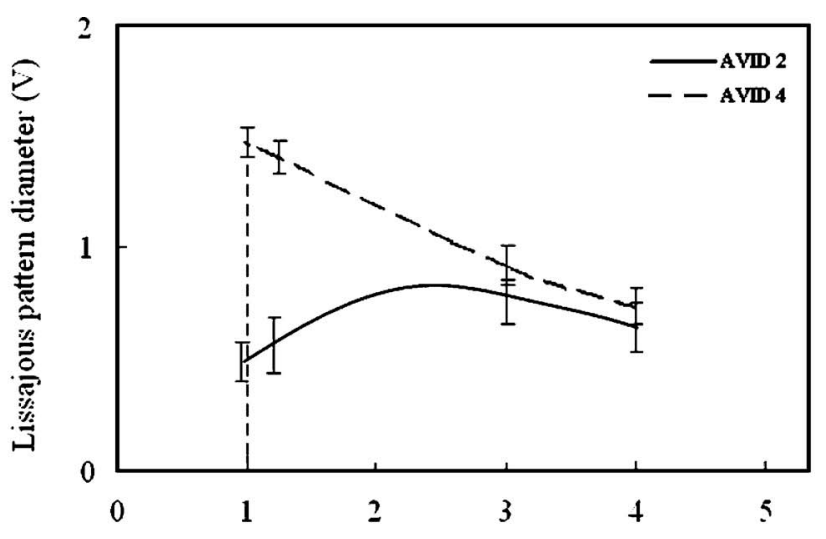

intensity ratio for outgoing beam $/$ and beam $2\left(I_{10} / I_{20}\right)$

(a)

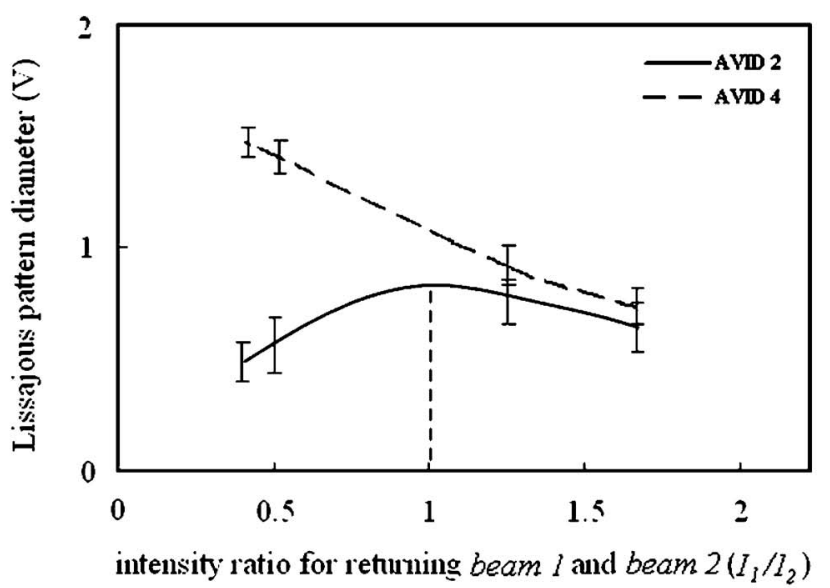

(b)

Fig. 6 Hard-disk gasket (partially specular surface) measurement results with different intensity ratios for beam 1 and beam 2 , expressed in terms of (a) outgoing beams and (b) returning beams. The error bars are the rms deviation of the data points from the diameter for the best-fit Lissajous circles and are enlarged 10 times.

that of the thin film disk and the weaker returning beam-1 intensity induced lower interference efficiency and resulted in a smaller Lissajous circle diameter, the optimum operating condition remains identical to that of the thin film disk measurement case. Testing data from these two specimens, one with a specular surface and the other with a slightly diffusive surface, confirms the state of the two optimal conditions as theoretically predicted by Eqs. (6) and (9).

Considering the case of the diffusive specimen magnet, it deserves to be mentioned that the reflection from the diffusive specimen destroys the light-beam wavefront as well as the polarization state. A portion of the distorted wavefront was recollimated by the objective lens. Measuring through the objective lens, with and without the PBS2, leads to the conclusion that the reflectivity of the diffusive gasket is 0.3 and $87 \%$ of the returned light beam remains $p$-polarized. With this set of retrieved data, the returning beam- 1 intensity was determined to be $0.2114 \quad\left(=0.9^{2}\right.$ $\times 0.3 \times 0.87)$ times the outgoing beam- 1 intensity. Measurements performed under the same conditions in AVID 2 and AVID 4 configurations are shown in Fig. 7(a) and Fig. 7(b). Out of the many experiments performed using the 


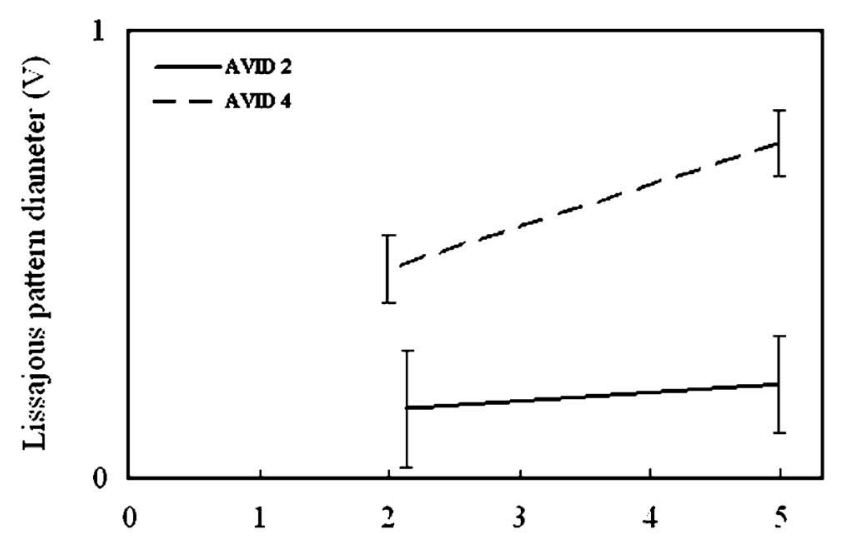

intensity ratio for outgoing beam 1 and beam $2\left(I_{10} / I_{20}\right)$

(a)

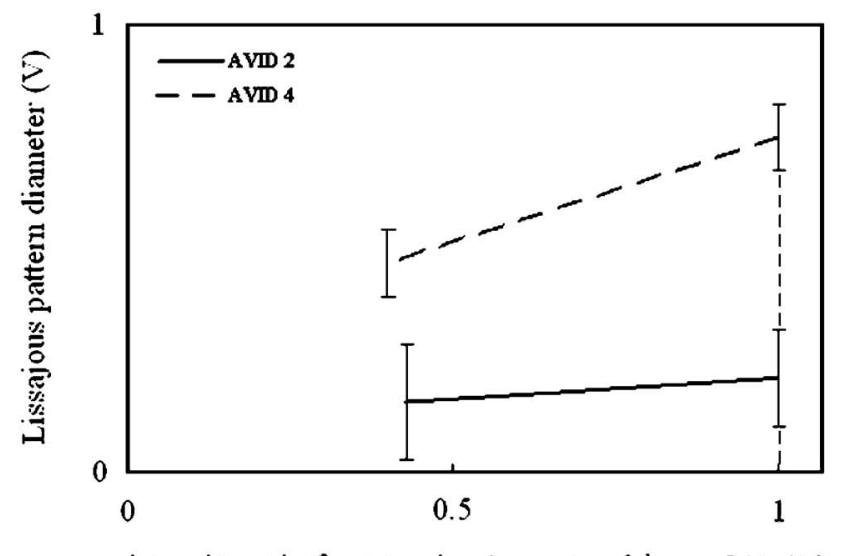

intensity ratio for returning beam 1 and beam $2\left(I_{1} / I_{2}\right)$

(b)

Fig. 7 Hard-disk magnet (diffusive surface) measurement results with different intensity ratios for beam 1 and beam 2 expressed in terms of (a) outgoing beams and (b) returning beams. The error bars are the rms deviation of the data points from the diameter for the best-fit Lissajous circles and are enlarged 10 times.

AVID 4 configuration, the best results were obtained at a 5:1 intensity ratio for $I_{10}$ versus $I_{20}$, which is equivalent to a $1: 1$ intensity ratio for $I_{1}$ versus $I_{2}$ for the AVID 2 configuration. More specifically, the testing results basically indicate that making $I_{1}$ and $I_{2}$ identical leads to an optimum metrology condition in both AVID 2 and AVID 4 configurations when a diffusive specimen is measured. However, this appears to conflict with the optimum operating condition predicted by Eq. (9).

It should be noted that on comparing the data in Fig. 7(a) and Fig. 7(b), the error bars of AVID 2 are seen to be longer than those of AVID 4. This difference can be attributed to the noise cancellation provided by the differential circuit adopted in AVID 4. Comparing the results shown, it is clear that AVID 4 attains its optimum operating point for the majority of the applications on keeping the two outgoing beams equal in intensity. Except for the case where the specimen surface becomes too diffusive, the optimum operating condition will change. When the relative intensities of the two outgoing beams are identical, it means that the optimum system operating point remains identical even for specimens possessing different optical properties. Thus, no adjustment is needed to optimize the experimental conditions. This result indicates that the AVID 4 configuration is more desirable than the AVID 2 configuration from a user's perspective. Nevertheless, the ability to adjust the polarization state of the laser beam before it hits the first polarization beamsplitter so as to vary the light-beam intensity ratio does provide a convenient approach to optimize the operating conditions for even wider applications. In fact, the optimum condition can be achieved by maximizing the size of the Lissajous circle pattern obtained while adjusting the intensity ratio, which automatically takes the wavefront and polarization into consideration. The design approach presented in this paper provides us with a way to optimize the design of circular polarization interferometers.

\section{Conclusions}

Two configurations of a microscope-based circular polarization interferometer developed for measuring the vibration of miniature specimens have been detailed. From the perspectives of the optical system designer and the user, the differences between the optimal operating conditions of these two configurations - a two-photodetector and a fourphotodetector circular-polarization interferometer-were examined in detail. It was noted that the difference between these two systems lies only in the number of photodetectors and in whether two polarizers or two cube-type polarization beamsplitters are used. Both theoretical and experimental results are detailed to demonstrate the strong association between the optoelectronic configuration and the signalprocessing algorithm adopted. It can be clearly seen that the optimum condition of a two-photodetector configuration will be obtained when the two returning beams achieve equal intensity. On the other hand, the four-photodetector circular polarization interferometer attains its optimum operating condition when the two outgoing light beams are identical before hitting the surface. This is valid for almost all specimen conditions except when the specimen surface is highly diffusive. It can also be shown that the optimum operating condition for the four-photodetector circularpolarization interferometer is identical to that of the twophotodetector interferometer when dealing with a highly diffusive specimen. These results indicate that, from a user's perspective, coupling a four-photodetector circularpolarization interferometer with the ability to adjust the outgoing light beam intensity provides us with a very versatile design configuration.

\section{Acknowledgments}

The authors would like to acknowledge the continuous and generous financial support of this research from the Ministry of Economic Affairs, Department of Industrial Technology of Taiwan, R.O.C., and the National Science Council of Taiwan, R.O.C., through grants 91-EC-17-A-05-S1-0017 and NSC 90-2323-B-002-007. In addition, the continuous support of the instrument team at AHEAD Optoelectronics, Inc., is acknowledged.

\section{References}

1. L. Y. Zhu and D. B. Bogy, "Head-disk spacing fluctuation due to disk topography in magnetic recording hard disk files," in Tribology and Mechanics of Magnetic Storage Systems, STLE Special Publication SP-26, pp. 160-167 (1989).

2. F. Durst, A. Melling, and J. H. Whitelaw, Principles and Practice of 
laser-Doppler anemometry, Academic Press, New York (1981).

3. L. E. Drain, The Laser Doppler Technique, Wiley, Norwich (1980).

4. T. A. Riener, A. C. Goding, and F. E. Talke, "Measurement of head/ disk spacing modulation using a two channel fiber optic laser Doppler vibrometer," IEEE Trans. Magn. 24(6), 2745-2747 (1988).

5. C. K. Lee and T. W. Wu, "Differential laser interferometer for nanometer displacement measurements," AIAA J. 33(9), 1675-1680 (1995).

6. C. K. Lee, W. W. Chiang, D. W. Meyer, U. V. Nayak, T. C. O'Sullivan, and T. W. Wu, "Miniature differential laser interferometer/vibrometer for optical glide and other storage applications," IBM Tech. Discl. Bull. 37(5), 185-187 (1994).

7. C. K. Lee and T. W. Wu, "New differential laser interferometer/ vibrometer for nanometer structural displacement/vibration measurements," IBM Tech. Discl. Bull. 35(1A), 189-195 (1992).

8. C. K. Lee, G. Y. Wu, Thomas C. T. Teng, W. J. Wu, C. T. Lin, W. H. Hsiao, H. C. Shih, J. S. Wang, Sam S. C. Lin, Colin C. Lin, C. F. Lee, and Y. C. Lin, "A high performance Doppler interferometer for advanced optical storage systems," Jpn. J. Appl. Phys., Part 1 38(3B), 1730-1741 (1999).

9. C. K. Lee, G. Y. Wu, W. J. Wu, K. S. Yeh, and C. F. Lee, "Interference apparatus for measuring absolute and differential motions of same or different testing surface," U.S. Patent No. 5,949,546 (1999).

10. K. Weir, A. W. Palmer, and K. T. V. Grattan, "Accurate measurement of small displacement using optical techniques," Int. J. Optoelectron. 9(6), 449-455 (1994).

11. X. Liu, W. Clegg, and B. Liu, "Ultra low head-disk spacing measurement using dual beam polarization interferometry," Opt. Laser Technol. 32(4), 287-291 (2000).

12. J. Y. Lee and D. C. Su, "Central fringe identification by phase quadrature interferometric technique and tunable laser-diode," Opt. Commun. 198(1), 333-337 (2001).

13. S. Inoué, Video Microscopy, Plenum, New York (1986)

14. E. Hecht, Optics, Addison-Wesley, New York (1998).

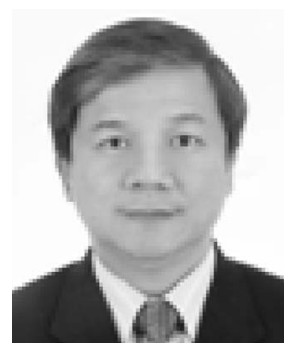

Chih-Kung Lee received his MS and $\mathrm{PhD}$ degrees from Cornell University in 1985 and 1987, respectively, with a major in theoretical and applied mechanics, and a minor in physics. Then he spent seven years at IBM Almaden Research Center in San Jose, California, as a research staff member and as staff to the laboratory director. Since 1994, he has been a professor at the Institute of Applied Mechanics, National Taiwan University. He has also held a concurrent position since 2002 as professor of the Department of Engineering Science and Ocean Engineering, National Taiwan University. His interests include microelectromechanical and nanotechnology systems, automation technology, design and construction of optoelectronic systems, optoelectronic and piezoelectric systems, precision metrology, and biochip systems.

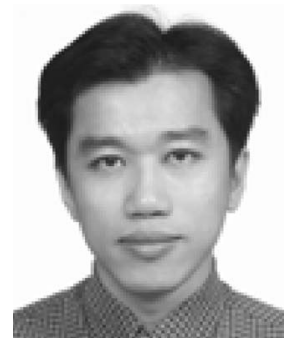

Wen-Jong Wu received his $\mathrm{PhD}$ from the Institute of Applied Mechanics, National Taiwan University, in 2002, and is now an assistant professor in the Department of Engineering Science and Ocean Engineering, National Taiwan University. His research interests are in system design and integration of precision metrology, smart sensor networks, and piezoelectric power devices.

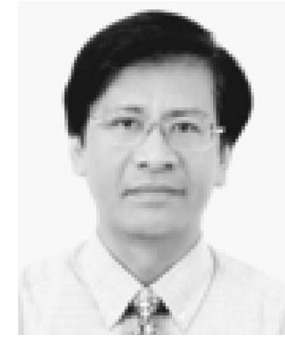

Giin-Yuan Wu received his PhD from the Institute of Applied Mechanics, National Taiwan University, in 1997. He is the general manager of Sunwave Optoelectronics, Inc. His research focuses on advanced laser vibrometers, LED spectrum testers, and color functional testers.

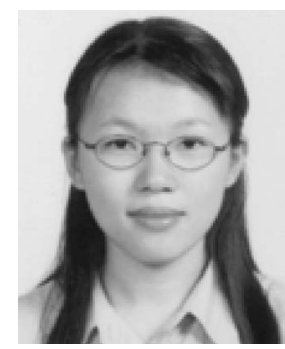

Chia-Ling Li received her bachelor's degree from the Department of Mechanical Engineering, National Taiwan University. She is pursuing a master's degree at the Institute of Applied Mechanics, National Taiwan University. Her research interests are optical system design, diode laser modulation, and interferometry.

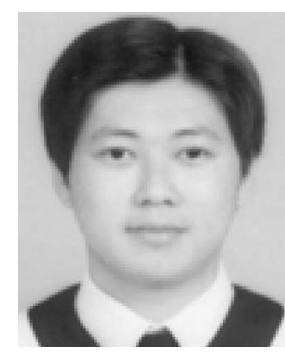

Zeng-De Chen received his bachelor's degree from the Department of Mechanical Engineering, National Taiwan University. $\mathrm{He}$ is pursuing a master's degree at the Institute of Applied Mechanics, National Taiwan University. His research interests are optical design, electronic circuit design, and digital signal processing (DSA).

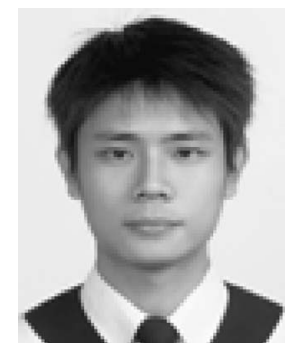

Jau-Yu Chen received his bachelor's degree from the Department of Bio-Industrial Mechatronics Engineering, National Taiwan University, in 2002. He is now pursuing a master's degree at the Institute of Applied Mechanics, National Taiwan University. His research interests include optical system design, optical metrology technology, and embedded systems. 